\title{
LOS TRATAMIENTOS Y SUS FÓRMULAS EN EL ESPAÑOL DE GUINEA ECUATORIAL: UN ESTUDIO BASADO EN la obra de JUAN Tomás Ávila LaUREL
}

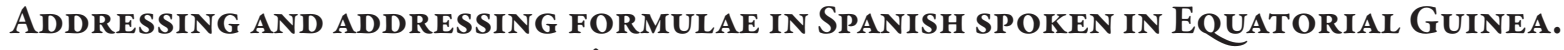 \\ A STUdY Based ON JUAN TOMás Ávila LAUREL's WORK
}

LES FORMULES D'ADRESSE (TUTOIEMENT ET VOUVOIEMENT) DANS L'ESPAGNOL DE GUINÉE EQUATORIALE: UNE etude basée sur l'oeuvre de Juan Tomás AVila LaUrel

\author{
Issacar Nguendjo \\ Doctor, Universidad Complutense de \\ Madrid. Profesor titular de Lengua y \\ Lingüística Españolas y coordinador \\ del Máster de Estudios Hispánicos \\ en el Departamento de Lenguas \\ Extranjeras Aplicadas, Universidad \\ de Dschang, Camerún. \\ Université de Dschang, Faculté des \\ Lettres et Sciences Humaines, BP 49, \\ Dschang, Cameroun. \\ flsh@univ-dschang.org
}

\begin{abstract}
RESUMEN
Los tratamientos y sus fórmulas presentan una configuración y una distribución muy complejas en el paisaje hispanófono en general. Sin embargo, como pasa muchas veces en los trabajos de lingüística española, se suelen limitar los estudios al español peninsular e hispanoamericano. El presente artículo analiza la cuestión de las fórmulas de tratamiento en otro espacio con características específicas, Guinea Ecuatorial, único país hispanófono de África subsahariana. Para ello, desde una aproximación metodológica inductiva, la reflexión se basa en un corpus múltiple, un cuento y dos novelas del autor guineano Juan Tomás Ávila Laurel, para poner de relieve el cruce de distribuciones, que sitúa el español de Guinea Ecuatorial fuera de la hispanofonía voseante por una parte, e indecisa en la distribución objetiva y sistemática entre el tuteo y el ustedeo (tú/vosotros/ustedes) tal como se presenta en España, por otra. Como hipótesis principal, planteamos la inexistencia, en las lenguas locales, de un pronombre personal (de segunda persona, con forma verbal correspondiente) que sirva de equivalente para el "usted" peninsular. Es ello un reflejo interferencial de las relaciones proxémicas en las lenguas y culturas locales que se plasma en la lengua española en tierras africanas.
\end{abstract}

Palabras clave: fórmulas de tratamiento, español de Guinea Ecuatorial, Juan Tomás Ávila Laurel

\begin{abstract}
Addressing and its formulae show a highly complex configuration and distribution throughout the Spanish-speaking landscape as a whole. However, as usual in Spanish language linguistics studies, works addressing this issue are commonly limited to Peninsular and Hispanic American Spanish. This paper examines the issue of addressing formulae at another specific featured place - Equatorial Guinea, the only Spanish-speaking country in sub-Saharan Africa. In order to do that, this reflection is based on a multiple corpus, a short story, and two novels by Guinean author Juan Tomás Ávila Laurel, following an inductive methodological approach. This intends to highlight the layout crossing situating Equatorial Guinea Spanish language outside of the voseo-using Hispanofonía on the one hand, and undecided between the objective and systematic distribution between tuteo and
\end{abstract}


ustedeo (I, you), as seen in Spain, on the other hand. As the main hypothesis, we put forward the non-existence, in native local languages, of a personal pronoun (second person, with a corresponding verbal form) to match the Peninsular usted form. This is an interferential reflection of proxemic relations between local languages and cultures that is observed in Spanish language in African lands.

Keywords: addressing formulae, Equatorial Guinea Spanish, Juan Tomás Ávila Laurel

\section{RESUMÉ}

Les formules d'adresse présentent aussi bien une configuration qu'une distribution très complexes dans le paysage hispanophone en général. Cependant, comme c'est le cas dans la plupart des questions de notre discipline, les études sont généralement limitées à l'espagnol péninsulaire et hispano-américain. Ainsi donc, cet article vise à analyser la question des formules de traitement dans un autre espace qui présente des caractéristiques spécifiques, à savoir la Guinée Equatoriale, seul pays hispanophone de l'Afrique subsaharienne. Pour ce faire, à partir d'une approche méthodologique inductive, la réflexion est basée sur un corpus multiple, un conte et deux romans de l'écrivain guinéen Juan Tomás Ávila Laurel, pour mettre en évidence le chevauchement des distributions, qui place l'espagnol de la Guinée équatoriale en dehors de l'hispanophonie voseante d'une part, et indécise dans la distribution objective et systématique entre le tutoiement et le vouvoiement (tu /vous/vous) tel que cela se présente en Espagne, d'autre part. L'hypothèse principale semble être l'absence dans les langues locales d'un pronom personnel (deuxième personne, avec une forme verbale correspondante) pour servir d'équivalent du vous péninsulaire. Un reflet interférentiel des relations proxémiques telles que structurées dans les langues et les cultures locales qui transparaît dans la langue espagnole en terre africaine.

Mots clés : formules d'adresse, espagnol de Guinée Equatoriale, Juan Tomás de Ávila Laurel 


\section{Introducción}

No cabe duda de que entre la lengua y la sociedad existe una relación muy estrecha, íntima e indisociable. Según Casalmiglia y Tusón, la lengua es un hecho social:

[...] el material lingüístico se pone al servicio de la construcción de la vida social, de forma variada y compleja en combinación con otros factores [...]; los elementos cognitivos, sociales y lingüísticos se articulan en la formación del discurso. Las lenguas viven en el discurso y a través de él (2012, pp. 2-3).

La lengua es tanto el reflejo como el vehículo de un haz de relaciones, de hechos, de costumbres, de estados propios a una sociedad y su cultura inherente. A partir de ahí, el acto comunicativo, es decir, dirigirse a otro(s), más allá del enunciado (con el contenido y la intención) que se emite por un enunciador a un(os) enunciatario(s) determinado(s), va cargado de otro tipo de datos de carácter proxémico, ${ }^{1}$ que informa sobre la relación que existe entre los interlocutores. Aquí es donde intervienen las llamadas fórmulas de tratamiento. Estas son las distintas opciones de pronombres y los cambios gramaticales, especialmente en la segunda persona tanto del singular como del plural, relacionados con el respeto, la confianza y la estructura social con las que nos dirigimos a otra(s) persona $(s){ }^{2}$ Norma Carricaburo afirma que "dada la amplitud territorial del conjunto de países que hablan la lengua española y las implicaciones socioculturales y psicosociológicas que presentan las fórmulas de tratamiento, el tema es sumamente complejo" (1997, p. 7). Para comprobar tal aserción, ella se propone estudiar la cuestión en el conjunto de los países del mundo hispanófono: la España centro peninsular e Hispanoamérica.

1 La proxemia se refiere a la distancia física o psicológica, a la relación de confianza, de autoridad o de poder que puede existir entre interlocutores, y que influye en la elección del pronombre de segunda persona a la hora del intercambio comunicativo.

2 Los títulos, como doctor, profesor, su majestad, excelencia, su señoría etc., también intervienen en la noción del tratamiento, aunque sin mayor incidencia gramatical. Sin embargo, no so n relevantes para el presente estudio.
Sin embargo, como desgraciadamente suele ocurrir en la gran mayoría de los trabajos de lingüística en general y en las cuestiones de lengua española en el mundo en particular, Guinea Ecuatorial suele ser ignorada de la hispanofonía, por motivos que no se pueden contemplar en el presente trabajo. Asimismo, nuestra reflexión en este artículo encuentra su justificación en dos argumentos principales: reforzar tal hipótesis de la complejidad del tema de las fórmulas de tratamiento en el mundo hispanófono en general y enfocar el estudio en el país centroafricano, único en su perfil "afrohispanobantú". ${ }^{3}$ Guinea Ecuatorial es un país cuya "especificidad [...] en términos de identidad está esencialmente basada en la combinación de identidades que corresponden a las culturas hispana y bantú" (Ambadiang, 2010, p. 50). Nuestro propósito, entonces, es analizar cómo se configura la noción de tratamiento en el español de Guinea Ecuatorial y cuáles son las fórmulas vigentes en este espacio. Para ello, desde una aproximación metodológica inductiva, basamos nuestro estudio y argumentación sobre tres novelas del autor ecuatoguineano Juan Tomás Ávila Laurel, como muestra de lo que se puede generalizar a toda la sociedad ecuatoguineana.

Juan Tomás Ávila Laurel se presenta como una de las figuras más representativas de la llamada "literatura popular" de Guinea Ecuatorial. Se trata de una colección que fue pensada para "ofrecer una literatura de corte popular que satisfaga las necesidades de un amplio sector de la población" (Gómez-Pimpollo, editora de la revista El Patio, citada en Laínez, 1999, p. 64). ${ }^{4}$ Dicha literatura

3 Un neologismo (Nguendjo, 2014) que se justifica por la particularidad de Guinea Ecuatorial como país africano, hispanófono y, esencialmente, bantú, a diferencia de otros territorios hispanizados del continente (El Sahara occidental, Ceuta y Melilla, Islas Canarias), cuyos perfiles no se pueden equiparar.

4 El proyecto fue lanzado por el Centro Cultural Hispano Guineano de Malabo en los años noventa, para fomentar la producción literaria en lengua española entre los guineanos, mediante su revista El Patio. De este proyecto salieron nombres hoy conocidos como Maximiliano Nkogo, José Siale Ndajngany, entre otros. 
floreció a finales de la década de los noventa y tiene como característica esencial la pintura costumbrista de la sociedad ecuatoguineana, riéndose de sus lacras y de sus mayales. Dicha descripción de hechos sociales y especialmente urbanos también se transparenta, en la lengua de expresión literaria, a través de los personajes y del autor-narrador. Asimismo, nos ofrece un material escrito que refleja fidedignamente la lengua española en su práctica entre los guineanos. Estas características nos han parecido muy interesantes y justifican la elección de nuestro corpus para apoyar una investigación con exigencias científicas, aportando datos ilustrativos documentados. La escritura laureliana es constante (por sus publicaciones desde los años 2000), espontánea, diversificada (en término de géneros literarios) y pictográfica.

También nos valemos de nuestra experiencia personal en nuestros intercambios con ciudadanos de dicho país. En un primer momento, recordamos las principales tendencias y los modos de funcio-

namiento de las fórmulas de tratamiento en España e Hispanoamérica; luego esbozamos un perfil sociolingüístico e histórico de Guinea Ecuatorial, y terminamos por una presentación concreta de cómo se estructura la noción de tratamiento en el español de Guinea Ecuatorial.

\section{Los pronombres y las formas verbales en la norma del español}

Hablar de las fórmulas de tratamiento en la orientación que nos interesa en este apartado significa considerar en sus detalles los pronombres personales y las formas verbales correspondientes a cada uno de ellos. Sin embargo, de todos los pronombres de los que disponga cada lengua, los de la segunda persona (del singular y del plural) merecen especial atención, ya que son los que están implicados en situaciones de intercambio directo. Un individuo (emisor o enunciador) que se dirige a otro(s) individuo(s) (receptor(es) o enunciatario(s)) necesita tanto un pronombre como una forma verbal adecuados para dar forma a su enunciado, de acuerdo con su relación proxémica con su(s) interlocutor(es).
La segunda persona del singular en el español peninsular moderno consta de dos pronombres personales, tú y usted, mientras que en el plural tenemos vosotros, vosotras y usted(es). Este último suele considerarse como la forma de tratamiento de respeto (por la edad, el rango social, al poder político, etc.), y el verbo adopta la forma verbal de tercera persona singular o plural eventualmente, mientras que el tú se usa en circunstancias menos formales, donde existe una relación de confianza, de familiaridad entre los interlocutores. Vosotros y vosotras aparecen, entonces, como fórmulas de tuteo colectivo, es decir, cuando uno se dirige a más de un interlocutor mediante el tratamiento de confianza (el tuteo). Individuos de clase similar o igual se pueden tratar de usted(es) en situaciones formales. Por ejemplo, entre los adversarios políticos en un debate televisivo, lo normal es que se traten mutuamente de usted(es).

También es cierto que en algunos contextos de relación vertical, es decir, en la que ambas partes de la interlocución no se ven en el mismo nivel de edad, de estatuto social o de poder, el sujeto que tenga más poder puede tratar de tú al interlocutor de menos poder, mientras que este último, por su parte, trata de usted al primero. A modo de ilustración, podemos considerar el caso de un profesor a su estudiante:

Profesor Tamo: ¿Has visto [tú] qué hora es? Siempre llegas tarde y entras sin llamar [tú]. ¿Crees [tú] que mi clase es un mercado? Alumno Musa: Discúlpeme [usted], Señor profesor. Usted nos dijo que no le interrumpiéramos cuando llegamos tarde. Le prometo [a usted] que esto no volverá a ocurrir.

Para sintetizar lo que precede, podemos aprovechar las palabras de Carricaburo cuando afirma que

[...] el eje horizontal puede ser recíproco para la solidaridad o confianza, cuando ambos se tutean (T-T), o recíproco para el poder o la diferencia, cuando ambos se tratan de usted (U-U). Cuando el eje es vertical, el polo

5 Experiencia en el aula, en clase de Análisis del discurso, a estudiantes de español del nivel 3, 11 de mayo de 2017 , Universidad de Dschang, Camerún. 
del tuteo o del voseo es el que está marcado por el menor poder (- poder) (1997, p. 9).

Esta norma peninsular coexiste en la actualidad con otro paradigma de pronombres personales de tratamiento en algunas zonas de Hispanoamérica. Se trata precisamente del pronombre vos, que en muchas partes de la América voseante (Río de la Plata, Pacífico sur, América central y ecuatorial) sustituye al tú peninsular, o simplemente cohabita con él. En partes de Venezuela, por ejemplo, "el tú suele ser utilizado como fórmula de tratamiento intermedio, o sea, más formal que vos y menos formal que usted" (Carricaburo, 1997, p. 42). Y en tal contexto, la forma ustedes se convierte en el único pronombre para referirse a la segunda persona del plural. Es decir, sustituye tanto al vosotro(a)s, como al ustedes peninsulares, aunque muy a menudo con el significado de vosotros.

Vos es un pronombre que presenta un alto grado de complejidad en su uso. En efecto, no manifiesta homogeneidad ni en la forma verbal con la que concuerda, ni en las circunstancias y los contextos, ni en las áreas dialectales en los que se usa. En este sentido, José Pedro Rona (1967) cataloga el voseo en cuatro tipos, basados en las desinencias verbales en el presente de indicativo, y que reflejamos en la tabla 1 .

Una observación estructural de las desinencias verbales (del presente de indicativo) en las que se fundamenta tal categorización nos proporciona argumentos para defender la hipótesis de que vos, aunque compita con tú, parece proceder de vosotros. De hecho, excepto el voseo de tipo $\mathrm{IV}$, que parece coincidir con las formas verbales

Tabla 1 Tipología de voseos

\begin{tabular}{lrll}
\hline \multicolumn{3}{c}{ Conjugaciones } \\
\hline Tipo de voseo & \multicolumn{1}{c}{ 1. $^{a}$} & \multicolumn{1}{c}{ 2." $^{\text {a }}$} & \multicolumn{1}{c}{ 3. $^{a}$} \\
Voseo I & cant-áis & beb-éis & viv-ís \\
Voseo II & cant-áis & beb-ís & viv-ís \\
Voseo III & cant-ás & beb-és & viv-ís \\
Voseo IV & cant-as & beb-es & viv-es \\
\hline
\end{tabular}

Fuente: Elaboración propia. correspondientes a tú, las demás, por la posición de la sílaba tónica (tilde) del verbo, apuntan hacia un cruce con el pronombre vosotros $(a) s$, como observa Rafael Lapesa:

\section{[...] vos concuerda ordinariamente con formas verba- les que en su origen fueron de plural: imperativos sin - $d$ final (cantá, poné, vení) usados en España hasta el siglo XVII, y presentes de indicativo sin diptongo en la desinencia (andás, tenés, saliś) desechados aquí duran- te el siglo XVI (1981, pp. 580-581).}

A mododeconclusión parcial paraeste primerapartado, podemos mencionar que, en lo que se refiere a las fórmulas de tratamiento, los estudios realizados sobre la cuestión contemplan dos principales sistemas: uno en la península (tuteo/ustedeo) y otro en Hispanoamérica (tuteo/ustedeo/voseo).

\section{Breve perfil linguiístico de Guinea Ecuatorial}

La República de Guinea Ecuatorial presenta un perfil sociolingüístico a la vez distinto y peculiar dentro del mundo hispanófono. En efecto, es el único país del África subsahariana que pertenece a esta comunidad. A consecuencia del Tratado de Pardo (en 1778), pasó a ser colonia española hasta 1968, cuando obtuvo su independencia.

Guinea Ecuatorial "es un crisol de lenguas donde el español no es la lengua materna de ninguno de sus hablantes" (Quilis y Casado-Fresnillo, 1995, p. 35), lo que les confiere un perfil plurilingüe con las interferencias inherentes a este tipo de entornos. A las tres lenguas oficiales, que son el español, el francés y el portugués, ${ }^{6}$ se suman sendas lenguas maternas, como el fang, el fadambú o annobonés ${ }^{7}$

6 Guinea Ecuatorial adoptó el francés como segunda lengua oficial e ingresó en la francofonía desde 1998, para integrar la Comunidad Económica y Monetaria de África Central (CEMAC), donde todos los demás miembros son francófonos. Por intereses de misma índole, en 2007, el presidente Obiang Nguema declaró el portugués como tercera lengua oficial del país. Sin embargo, hay que reconocer que el uso efectivo de ambas lenguas es muy poco apreciable entre los ecuatoguineanos.

7 Es la lengua materna del autor Juan Tomás Ávila Laurel. 
(un criollo de base lusitana), el bubi, el benga, el bujeba, el kombe, el balengue, etc., todas de estirpe bantú. Luego, hay que mencionar el pichí, ${ }^{8}$ un pidgin que Juan Tomás Ávila Laurel llama "pasaporte lingüístico" (2002b, p. 15) o "lengua de ciudad" (Ávila Laurel, 2002b, p. 25) de Malabo (isla de Bioko), en particular, y de Guinea Ecuatorial, en general.

John Lipski define el pidgin como "lenguaje reducido - que carece de toda inflexión, conjugación, concordancia, y complejidad sintáctica- $-[. .$. adoptado por los miembros de distintos grupos lingüísticos para facilitar la comunicación básica entre sí” (s. f.). Una definición que no comparten otros estudiosos como Kofi Yakpo, para quien

[...] la dominación colonial española fomentó una actitud negativa hacia los lenguajes africanos en general. Las similitudes superficiales entre el vocabulario del pichi y del inglés, y la supuesta simplificación de las estructuras del inglés que los observadores europeos creyeron encontrar en una lengua que no dominaban, combinadas con la idea racista sobre la superioridad de los idiomas europeos, ocasionaron que los administradores y los misioneros españoles consideraran al pichi como una modalidad empobrecida y desgajada del inglés (2009, p. 11).

En tal contexto de pluralidad y de heterogeneidad sociolingüística, es común que las lenguas y las culturas interfieran unas sobre otras. Asimismo, hemos notado que tanto el pichí como las lenguas maternas de los ecuatoguineanos comparten algunos aspectos que pueden influir en la lengua española en este país, precisamente en lo que se refiere a las fórmulas de tratamiento. Por ejemplo, dichas lenguas cuentan con un sistema verbal muy simplificado, desprovisto de flexiones, lo que hace que los tiempos verbales, los pronombres sujetos y objetos no se reflejen en las terminaciones verbales como se produce en el castellano. A modo de

8 "El pichí también es conocido con los nombres de 'Fernando Po creole English' (cf. Gordon, 2005), 'Fernando Po krio' (cf. e.g. Berry, 1970; Holm, 1989), 'Fernando Po pidgin English' (cf. e.g. Holm, 1989), 'pidgin' (Morgades, 2004), 'broken English' (e.g. De Zarco, 1938) y 'pichinglis' (e.g. Lipski, 1992)” (citado por Yakpo, 2009, pp. 9-10). ejemplo, podemos aprovechar las siguientes oraciones del pichí, a las que podríamos realizar cualquier transformación o sustitución de pronombres, de tiempo, de modo o de aspecto, sin que los verbos (en negrita) sufrieran alguna alteración:

If yù want, à fit sel yu mi hos. (Si quieres, puedo venderte mi casa) $)^{9}$ (Yakpo, 2009, p. 95).

Nà de à de wan hi ya à no fit du no natin. (Es allá donde estuve (por) un año, no pude hacer nada) (Yakpo, 2009, p. 96).

También podemos notar que el paradigma de los pronombres personales en dichas lenguas dista del que es vigente en el español tanto peninsular como hispanoamericano. Por ejemplo, el pichí sólo consta de dos pronombres personales para la segunda persona: yù para el singular y ùna/ùnu para el plural. Yakpo (2009, p. 86) subraya que las formas ùna/ünu son invariables a lo largo de todo el paradigma; y se emplean sin distinción de significado, aunque ùna es más común. Equivalen al vosotro(a)s, del castellano, que por necesidad se puede extender al ustedes.

En el caso de la lengua fang, la más importante por su número de hablantes y el estatuto del que ha gozado hasta la actualidad como lengua del Gobierno, ${ }^{10}$ Ndongo Esono (1956) no menciona ningún pronombre que equivalga a usted(es) del español peninsular, al mismo tiempo que da equivalencias de tú y de vosotros (sin mención explícita del femenino vosotras). Tampoco hay rastro del pronombre vos. Todo esto nos lleva a sospechar que simplemente no existen pronombres especiales en la lengua fang que corresponderían al tratamiento formal y de respeto, es decir, usted y ustedes. También se evidencia que Guinea

9 También se puede traducir en español por "si quiere (usted), puedo venderle mi casa”.

10 Nos referimos a una de las manifestaciones de la "fanguización" de la sociedad guineoecuatoriana, que algunos autores como Max Liniger-Goumaz (2013), Justo Bolekia Bolekà (2001 y 2011) o Fernando Muakuku (2006) describen en sus respectivos trabajos. 
Ecuatorial no forma parte la comunidad voseante del mundo hispanófono.

El panorama no es nada diferente en el annobonés, lengua materna de nuestro autor. A pesar de que clasificar el annobonés entre las lenguas bantúes presenta algunas reservas, ya que es un criollo, es decir, un pidgin hecho lengua materna de los nativos de la isla de Annobón, es indiscutible que todo pidgin se construye sobre una lengua lexificadora $^{11}$ (el portugués en este caso), y otra(s) lengua(s) que suele(n) imponer sus normas gramaticales. De ahí que podamos asegurar que la gramática annobonesa se asemeja a los fundamentos normativos que rigen las lenguas propias a los grupos étnicos de esta zona del mundo, que son de estirpe bantú.

Es importante recordar la trayectoria de la lengua española en Guinea Ecuatorial. El Tratado de Pardo, de 1776, que concedía Guinea Ecuatorial a la autoridad colonial española, no se tradujo en los actos hasta finales del siglo XVIII, cuando los misioneros españoles empezaron a instalarse en el territorio. La falta de interés por parte de España se puede explicar por la importantísima extensión de sus posesiones en América Latina. Y la independencia de Guinea Ecuatorial en 1968 coincidió con la llegada al poder de Macías Nguema, cuyo régimen no fue especialmente favorable a la apertura y a la promoción de la lengua española. Todo lo contrario: el español sufrió un retroceso a la vez cualitativo y cuantitativo, ya que solo se usaba en determinadas circunstancias, al mismo tiempo que el régimen intentó imponer el idioma fang. Solo en 1982, tres años después del "golpe de libertad", 12 el español recobró mayor prestigio y mejor estatuto como lengua oficial del país. Sin

11 La lengua lexificadora es aquella que nutre e inspira el patrimonio léxico del pidgin.

12 Con este término se maquilló el golpe de Estado que Teodoro Obiang Nguema, actual presidente del país, perpetró contra el régimen dictatorial de Macías Nguema. embargo, nunca llegó a ser la lengua principal de los guineanos. Como lo podemos leer en la introducción de Ekomo de María Nsue Angüe:

[...] en Guinea Ecuatorial [...] los nativos jamás han empleado el español comolengua materna. Para la comunicación interétnica se suele emplear el "pichi”" (inglés corrompido) en la isla de Bioko, mientras el fang desempeña este papel en el continente [...]. Sin embargo, se oye español por las calles [...], hablado entre guineanos [...]. Al ser una lengua artificial, el español guineano está ligeramente fosilizado (1985, pp. 9-10).

Con todos los datos que preceden, nos podemos preguntar qué impacto tienen tanto el perfil sociocultural como el historial lingüístico de los ecuatoguineanos en su manejo de la lengua española, una lengua colonial heredada, adoptada y adaptada. Es lo que pasamos a comprobar en el corpus que hemos manejado, enfocando el estudio en las fórmulas de tratamiento.

\section{La noción de tratamiento en el español de Guinea Ecuatorial}

Una constante en la obra literaria de Juan Tomás Ávila Laurel es la reafirmación de la no coincidencia entre la lengua de pensamiento de sus relatos y la lengua de expresión. El autor señala sin cesar la complejidad que surge a la hora de querer expresar realidades de su semioesfera etnocultural, mediante su lengua de escritura que es el castellano. En Arde el monte de noche (2009) podemos censar una veintena de enunciados sinónimos de "teníamos que hablar en una lengua que no era la nuestra” (p. 167). Una lengua que tiene una cosmovisión distinta de la española.

La noción de tratamiento ni se formula ni se estructura de la misma manera en ambas lenguas. Las lenguas guineanas en general y la del autor en particular no cuentan con pronombres personales específicos para expresar la cortesía, el respeto, el formalismo. Para manifestar el respeto hacia un jefe o persona mayor al dirigirse a él, se suele recurrir a otros sustantivos honoríficos o títulos. Por 
ejemplo, en la Guinea continental, los fang usan el título Nkukuma, ${ }^{13}$ con la forma verbal correspondiente al tú del español peninsular, lo que traduce la inexistencia de un equivalente para el pronombre usted (es) en nuestro contexto. La consecuencia de tal situación es el uso bastante confuso y poco sistematizado de los pronombres de segunda persona tanto del singular como del plural, que se mezclan y se alternan muy a menudo, incidiendo en la concordancia con las desinencias verbales.

En Rusia se va a Asamse (2002b) podemos observar, desde el inicio, cómo el autor-narrador se dirige en su relato a un lector virtual, mediante un trato de confianza, de familiaridad y de convivialidad: "ya sabes, lector, que de lo que se comió en la fiesta, Rusia no probó nada” (2002b, p. 13). Es evidente que el pronombre personal aquí es el tú, segunda persona del singular. Sin embargo, escasas páginas más adelante, se dirige al mismo interlocutor en las palabras siguientes:

[...] no me hagan caso; yo sabía de antes que las obras de misericordia son catorce pero a estas alturas del mundo, sería mucho pedir que me acordara de cada cual con su correspondiente ubicación en la tabla (2002b, p. 18).

Y posteriormente escribe: "la mayoría de los cotonús, fijaos en el plural, son mujeres maduritas y gordas" (2002b, p. 28). Este caso no parece ser una ocurrencia anecdótica o casual, ya que, en la novela Nadie tiene buena fama en este país (2002a), en algún intercambio entre dos personajes del relato, un hombre uniformado y una ciudadana común y corriente, el primero se dirige a la última, una desconocida, con los siguientes términos: "oiga, señora, ¿tú eres quien me ha puesto aquí?” (2002b, p. 44).

La situación no es nada distinta en otras lenguas de nuestro entorno como el fang, como se desprende de la siguiente conversación:

13 Carlos González Echegaray documenta dicha palabra con varias grafías: Nkukumaankukuma, o Cúcuma y la define como "indígena que ostenta la jefatura de un poblado o de una tribu” (1951, p. 108).

\begin{tabular}{ll}
\hline \multicolumn{1}{c}{ Fang } & \multicolumn{1}{c}{ Castellano } \\
\hline ¿Nza a lé? & ¿Quién Ilama? \\
Pedro. & Pedro. \\
Zoheñe na a ñihe (ñihi). & Dígale que entre. \\
Ma so a yahan na ua. & He venido a despedirme de ti. \\
¿Ye uakeemu? & ¿Se marcha usted hoy? \\
¿Ye onsoengoanzanzu? & ¿Volverás en el mes que viene? \\
Aa, ma ye so. & Sí; volveré. \\
Mvé o chuhaké. & Bueno, buen viaje. \\
Abora, akiba & Muchas gracias (Ndongo Esono, 1956, p. 45). \\
\hline
\end{tabular}

Podemos apreciar cómo, en la misma situación de intercambio, donde no hay modificación en la relación de proxemia, la versión española vacila entre el tuteo y el ustedeo, mientras que en fang solo se usa el tuteo $(u a=$ tú $)$.

Los extractos que preceden demuestran con claridad un solapamiento entre el tuteo y el ustedeo, con la consecuencia de que todos los pronombres de segunda persona pueden ser válidos, si consideramos que vosotros es forma de tuteo para dirigirse a más de una persona. Será exactamente lo que se produce en la cultura guineana, donde las relaciones proxémicas, aunque distingan entre cercanía y distancia, no cuentan con pronombres y formas verbales específicas para marcar la familiaridad o el respeto. Dicha configuración influye y se manifiesta en el castellano local. Los pronombres de segunda persona se cruzan a menudo, y pueden sustituirse de modo recíproco en la(s) lengua(s) de pensamiento, la del autor-narrador, contrario a lo que ocurre en la lengua española, donde se distinguen con criterios pertinentes. Las lenguas bantúes, mayoritarias en nuestro entorno de estudio, parecen pues estructurar los pronombres personales de segunda persona en un paradigma de distribución complementaria o libre, ${ }^{14}$ donde no existen los pronombres usted y ustedes.

En reiteradas ocasiones, Ávila Laurel (2009, pp. 49, 108) quiere distinguir con claridad entre "lengua de

14 Usamos esta terminología por analogía con la teoría fonológica sobre la distribución y el uso de los fonemas y de sus alófonos (véase Iribarren, 2009, p. 188). 
la gente de mi isla" o "lengua de la gente del mar" (que sin ninguna duda es el fadambú, lengua de la isla de Annobón, aunque no lo diga explícitamente) y el español, lengua del relato. En efecto, dicha narración empieza por una canción típica y ritual ${ }^{15}$ en el pueblo annobonés; una canción que acompaña el estreno de un cayuco:

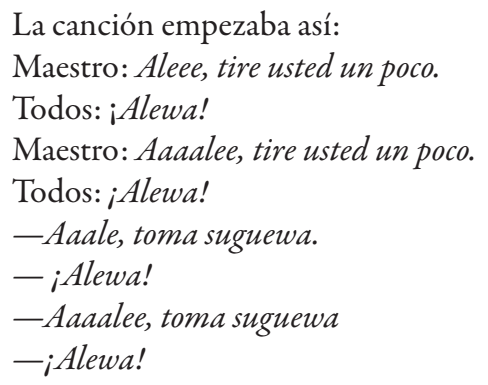

Y para explicar la canción, afirma que

[...] lo de "tire usted un poco" también podía ser "tirad vosotros un poco", o "tiren ustedes un poco", o "tiren de él un poco", o "tirad de él un poco”. ¿Sabéis por qué podía ser cualquiera de las versiones? Porque la lengua en la que se dice esto no tiene "usted", pero el "maestro" que dirige a "todos" se dirige a ellos con respeto, como si les tratara de usted; y es porque les ruega respetuosamente que tiren de algo (Ávila Laurel, 2009, p. 11).

\section{Conclusiones e implicaciones}

A modo de conclusión de esta reflexión sobre la distribución y el uso de las fórmulas de tratamiento en el español de Guinea Ecuatorial en general y en la obra de Ávila Laurel en particular, podemos afirmar que nuestro contexto de estudio presenta un panorama muy peculiar dentro del mundo hispánico. La situación de Guinea Ecuatorial sobre la cuestión no se asimila ni a la de España, ni a la de Hispanoamérica, principales escenarios de los estudios anteriores sobre la cuestión. El español de Guinea Ecuatorial está marcado por influencias heterolingüísticas, como consecuencia de su encuentro con otros idiomas, especialmente las

15 Nos referimos aquí a todo lo que gira en torno a la fabricación y el estreno del cayuco, un bien de alto valor simbólico en el paisaje sociocultural del pueblo isleño de Annobón. lenguas bantúes y el pichí. El castellano es aquí la lengua de expresión de ideas, pensamientos, hechos y costumbres concebidos desde lenguas con disposiciones culturales distintas, con lo que lo escrito o lo dicho parece ser una traducción, como lo afirma nuestro autor: "en mi lengua es una cosa que se puede traducir así [...]” (Ávila Laurel, 2009, p. 162).

Las conclusiones concretas a las que llegamos son las siguientes:

1. Guinea Ecuatorial no forma parte de los pueblos voseantes, ya que no se usa el pronombre vos ni en el habla ni en la literatura.

2. Tanto las lenguas guineanas de clase bantú como el pichí no cuentan con pronombres personales equivalentes a usted o ustedes. Esta situación se infiere del español local, provocando una redistribución de las únicas fórmulas de tuteo existentes (tú y vosotros/vosotras), en la que el hispanohablante ecuatoguineano puede sustituir recíprocamente el tú por el usted, el vosotros por el ustedes, ${ }^{16}$ dando lugar a muchos cruces y discordancias entre pronombre y desinencia verbal de número.

3. Tanto en las lenguas bantúes como en el pichí, los verbos conjugados no se identifican por las desinencias verbales de tiempo, de modo, o de número, lo que interfiere a menudo en el uso de la lengua española, en la medida en que dificulta la deducción objetiva del pronombre con los que deben concordar. La principal consecuencia de tal configuración es, una vez

16 También hemos registrado en la lengua hablada muchos casos de solapamiento entre usted y vosotros, que se podría achacar a la influencia de la lengua francesa, ya que el error es muy común entre los hispanizantes francófonos (en español como lengua extranjera - ELE-) como los de Camerún, donde escuchamos frases como: "Señor, he aprobado vuestra asignatura"; "Le he enviado el documento en vuestro correo electrónico" o "Usted habéis dicho que no tendríamos clases esta semana”. En francés, el pronombre vous se puede reemplazar tanto por vosotros como por usted, o ustedes; de ahí la interferencia. 
más, el cruce, el solapamiento y la confusión entre todos los pronombres de segunda persona, que parecen relacionarse en un paradigma de distribución combinatoria o libre.

4. Las discrepancias de distribución y de uso de las fórmulas de tratamiento entre las lenguas de Guinea Ecuatorial y el castellano, con las interferencias mencionadas más arriba, constituyen un punto delicado tanto en el aula de enseñanza del español como lengua extranjera, como en la traducción de textos entre las lenguas española, francesa e inglesa, por ejemplo.

En definitiva, la lengua,

[... como materia primera del discurso, ofrece a quienes la usan una serie de opciones (fónicas, gráficas, morfosintácticas y léxicas) de entre las cuales hay que elegir en el momento de (inter)actuar discursivamente. Esa elección, sujeta o no a un control consciente, se realiza de acuerdo con unos parámetros contextuales que incluyen la situación, los propósitos de quien la realiza y las características de los destinatarios, entre otros. Estos parámetros son de tipo cognitivo y sociocultural, son dinámicos y pueden estar sujetos a revisión, negociación y cambio (Casalmiglia y Tusón, 2012, p. 1).

\section{Referencias}

Ambadiang, T. (2010). Escrituras intersticiales y dinámicas de la alteridad: el "problema" de la lengua en la literatura negroafricana escrita en español. En L. Miampika y P. Arroyo (Eds.), De Guinea Ecuatorial a las literaturas hispanoafricanas (pp. 41-64). Madrid: Verbum.

Ávila Laurel, J. T. (2002a). Nadie tiene buena fama en este país. Avila: Malamba.

Ávila Laurel, J. T. (2002b). Rusia se va a Asamse. Ávila: Malamba.

Ávila Laurel, J. T. (2009). Arde el monte de noche. Madrid: Calambur.

Bolekia Bolekà, J. (2001). Lenguas y poder en África. Madrid: Mundo Negro.
Bolekia Bolekà, J. (2011). Estado y poder en Guinea Ecuatorial. Recuperado de http://www.angelfire.com/sk2/ guineaecuatorial/poder.htm.

Carricaburo, N. (1997). Las fórmulas de tratamiento en el español actual. Madrid: Cuadernos de lengua española.

Casalmiglia, H. y Tusón, A. (2012). Las cosas del decir. Manual de análisis del discurso. Barcelona: Ariel.

González Echegaray, C. (1951). Notas sobre el español en África ecuatorial. Revista de Filología Española, 35, 106-108.

Iribarren, M. C. (2009). Fonética y fonología españolas. Madrid: Síntesis.

Laínez, J. C. (1999, mayo de). Narrativa popular guineoecuariana: Rusia se va a Asamse, de Juan Tomás Ávila Laurel. El Patio. La revista de la cultura hispano-guineana, (63), 61-64. Recuperado de http:// bibliotecadigital.aecid.es/bibliodig/pub_aecid/es/ catalogo_imagenes/grupo.cmd?path $=1015315$

Lapesa, R. (1981). Historia de la lengua española. Madrid: Gredos.

Liniger-Goumaz, M. (2013). Guinée Équatoriale. Un demisiècle de terreur et de pillage. París: L'Harmattan.

Lipski J. (s. f.). Las lenguas criollas (afro)ibéricas: estado de la cuestión. Recuperado de http://www.csub.edu/ tfernandez_ulloa/hle/lipski-lenguas $\% 20$ criollas $\% 20$ afroibericas.pdf.

Muakuku Rondo Icambo, F. (2006). Conflictos étnicos y gobernabilidad: Guinea Ecuatorial. Barcelona: Carena.

Ndongo Esono, S. (1956). Gramática pamue. Madrid: Instituto de Estudios Africanos, Consejo Superior de Investigaciones Científicas.

Nguendjo, I. (2014). Contactos lingüisticos afrohispanobantúes. Reflexiones varias (1). Saarbrucken: Editorial Académica Española.

Nsue Angüe, M. (1985). Ekomo. Madrid: UNED.

Quilis, A. y Casado-Fresnillo, C. (1995). La lengua española en Guinea Ecuatorial. Madrid: UNED.

Rona, J. P. (1967). Geografía y morfología del voseo. Porto Alegre: Pontificia Universidad Católica.

Yakpo, K. (2009). Gramática del pichí. Barcelona: CEIBA y Centros Culturales Españoles de Guinea Ecuatorial.

How to reference this article: Nguendjo, I. (2018). Los tratamientos y sus fórmulas en el español de Guinea Ecuatorial: un estudio basado en la obra de Juan Tomás Ávila Laurel. Íkala, Revista de Lenguaje y Cultura, 23(3), 535-544. DOI: 10.17533/udea.ikala.v23n03a09 